\title{
Combining Fluorescence Microscopy with Electron Spectroscopic Imaging to Study the Morphology and Composition of Substructures within the Mammalian Cell Nucleus
}

\author{
M. J. Kruhlak*, M. J. Hendzel**, and D. P. Bazett-Jones*** \\ * Experimental Immunology Branch, National Institutes of Health, Bethesda, MD, USA, 20892 \\ ** Oncology, University of Alberta, Cross Cancer Institute, Edmonton, AB, T6G 1Z2, Canada. \\ *** Programme in Cell Biology, Hospital for Sick Children, Toronto, ON, M5G 1X8, Canada.
}

The mammalian cell nucleus contains multiple substructures, including compartments, domains, and bodies [1]. The structural compartmentalization of the nucleus is thought to provide a functional basis for regulating gene metabolism [2]. Studying the architecture of the nucleus relies heavily on microscopy-based techniques and involves examining the environment in which substructures are immersed, how the distribution and assembly of substructures is regulated, and whether different substructures exhibit distinct morphologies and composition.

We took advantage of numerous forms of microscopy to examine the architecture of the nucleus. Primarily, we utilized fluorescence microscopy to provide a spatial map, with desired temporal resolution, and combined the results with the elemental mapping capabilities of electron spectroscopic imaging (ESI) in the energy-filtering transmission electron microscope [3]. Using this correlative fluorescence and ESI technique, we compared the composition and morphology of well-known promyelocytic leukemia protein (PML) containing nuclear bodies [4] with glucocorticoid receptor (GR) containing bodies and the recently described matrix associated deacetylase (MAD) bodies [5]. We were able to generate phosphorus and nitrogen elementenhanced maps to compare the phosphorus to nitrogen ratios of components within the particular nuclear substructures. The comparison of $\mathrm{P} / \mathrm{N}$ ratios allowed us to distinguish primarily proteinbased structures from nucleic-acid containing structures. Using immunofluorescence microscopy, we found that the different structures harbor distinct sets of proteins, and thereby are compositionally different with respect to their specific molecular constituents, but are very much similar in morphology and size. When examined by ESI, to generate $\mathrm{P}$ and $\mathrm{N}$ elemental maps, we found that MAD bodies resemble PML bodies, at least during some stage in their existence. Both are nitrogen-rich and phosphorus-poor, and are protein-based. GR-containing bodies, however, contain phosphorus-rich fibers that permeate throughout the structure. The nature of the phosphorus-rich fibers within GR-containing structures is unclear, but based on $\mathrm{P} / \mathrm{N}$ ratios, most likely represent nucleic acid. By serial sectioning, we found the morphology and composition of the GR-containing bodies to be consistent throughout the structure. We did find phosphorus-rich fibers at the periphery of PML bodies, as previously reported [6]. Thus, GR-containing bodies are more intimately associated with nucleic acid-based structures than are PML or MAD bodies. We conclude that these seemingly similar subnuclear structures observed by fluorescence microscopy are distinct in biochemical composition, not just in their protein constituent profiles. Overall, the ability to analyze the elemental composition of nuclear substructures provides valuable clues to possible functional roles that the architecture of the nucleus may have in regulating gene metabolism. 
[1] A. I. Lamond and W. C. Earnshaw, Science. 280 (1998) 547.

[2] M. Dundr and T. Misteli, Biochem J. 356 (2001) 297.

[3] D. P. Bazett-Jones and M. J. Hendzel, Methods.17 (1999) 188.

[4] K. L. Borden, Mol. Biol. Cell. 22 (2002) 5259.

[5] M. Downes et al., Proc. Natl. Acad. Sci. USA. 97 (2000) 10330.

[6] F. M. Boisvert et al., J. Cell Biol. 148 (2000) 283.

[7] This research was supported by operation grants from the Canadian Institutes of Health Research.We gratefully acknowledge Ying Ren and Michael Schoel for their excellent technical support.

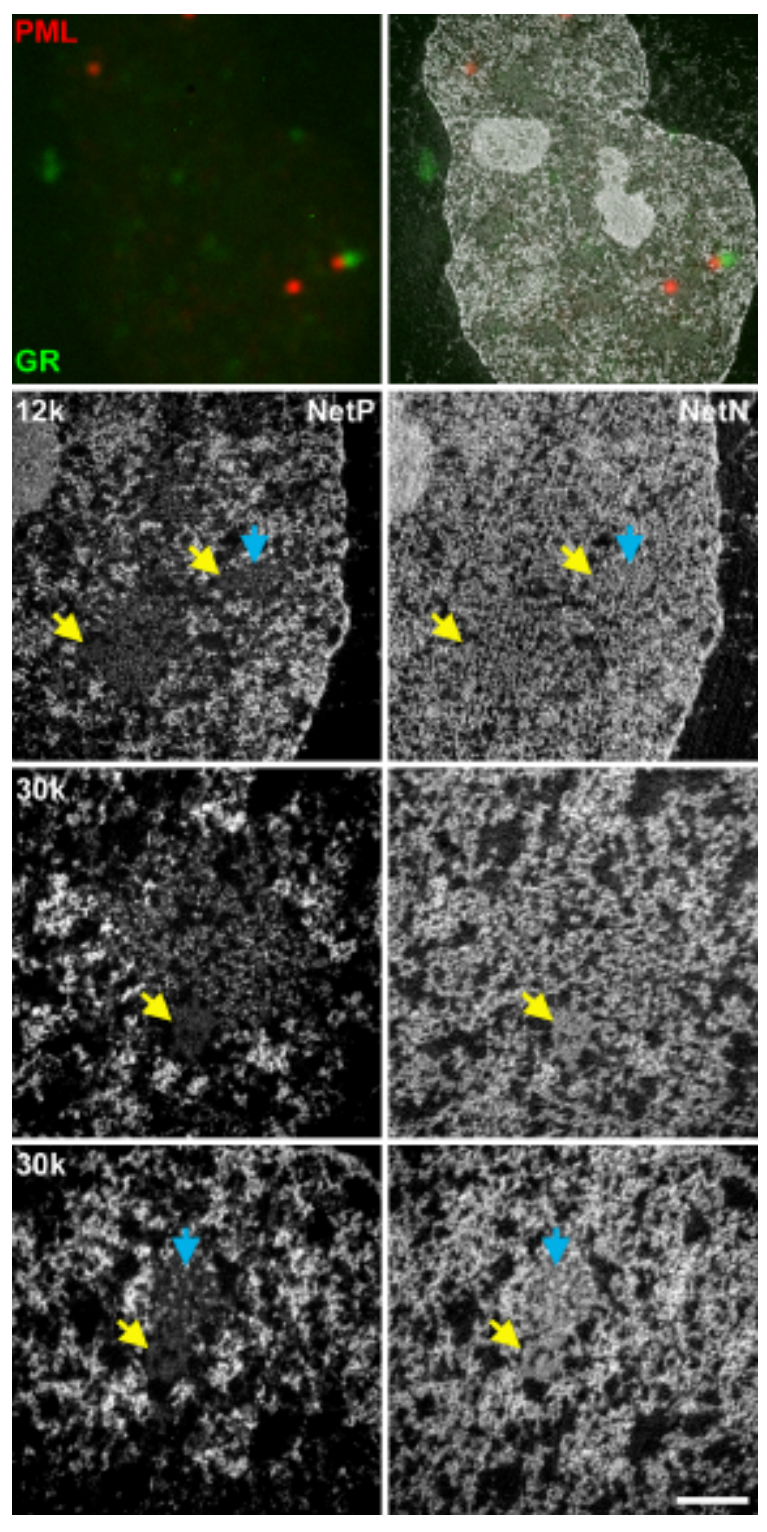

Fig 1. Correlative fluorescence and ESI images of PML and GR-containing bodies. Fluorescence image of PML (red) and GR (green) (top left panel). Net phosphorus (Net P) and nitrogen (Net N) enhanced maps (left and right columns, respectively) at 12 000x (12k) and $30000 \mathrm{x}(30 \mathrm{k})$ magnification. Yellow arrows denote PML bodies and blue arrow denotes GR body. Scale bar represents $\sim 400 \mathrm{~nm}$. 